\title{
Spectrum of non motor symptoms (NMS) in Parkinson's disease patients and correlating them with the severity of motor symptoms- a South Indian study
}

\begin{abstract}
Introduction: Non motor symptoms (NMS) impair the quality of life, delayed recognition of these symptoms will lead to disability and increase cost of care in PD patients.

Objectives: To study the spectrum of NMS in terms of their prevalence and frequency in a South Indian cohort of Parkinson's disease (PD)patients by using the NMSQ(Non motor symptom questionnaire), and to correlate the NMSQ-T(total score) with motor severity (UPDRS III) and to the (H\&Y) stage of disease.

Materials and methods: Patients presenting with features suggestive of Parkinson's disease to the Neurology OPD were recruited in the study. This was an observational and one time point study conducted at Narayana medical college and hospital during the period of 1 1/2 year from January 2015 to May 2016.

Results: The common NMS were constipation and anxiety followed by urgency of bladder. Comparison of NMSQ-T score with the UPDRS III and H\&Y staging demonstrated a significant statistical correlation between non-motor symptoms and motor severity and H\&Y staging of the disease.

Conclusion: Prevalence and frequency of NMS found to have significant association with motor severity and stage (H\&Y) of the disease in Parkinson's patients. NMS like incomplete bladder emptying, inability to participate in sex were having more significant statistical correlation than the rest of the symptoms. Further studies are required with large samples to confirm the predictability of motor severity of the disease by NMS.
\end{abstract}

Keywords: nonmotor symptoms, parkinson disease, UPDRS-III, H\&Y staging

\section{Introduction}

Parkinson's disease (PD) is one of the most common neurodegenerative movement disorder, characterized by bradykinesia, tremor, rigidity and postural reflexes derangement. It is a disease of elderly age group but the younger age is also not immune to the disease. Along with the motor manifestations Parkinson's disease is also associated with the nonmotor symptoms (NMS) which are not well recognized. The early identification of the NMS is essential to improve the quality of life in the affected patients. The emergence of the NMS during the natural history of the disease cannot be clearly delineated. NMS may be complained at the time of diagnosis ${ }^{1}$ or they may predate the disease diagnosis or they may present late in the disease course. Thus the knowledge of the nonmotor features along the side of motor symptoms can help in better management of Parkinson's disease on a whole.

NMS manifest with the involvement of non dopaminergic pathways in brain, and their response to the classical antiparkinsonian drugs like levodopa is also limited. ${ }^{2}$ Moreover the burden of NMS is found to be more on the PD patients who are on treatment. Disease duration and advanced age also can affect the NMS frequency and severity. ${ }^{3,4}$ Urinary symptoms, insomnia are the commonest among the NMS along with constipation, depression, memory and sexual problems. ${ }^{5,6}$
Volume 9 Issue 2 - 2019

\author{
NS Sampath kumar,' Ganesh Vallampalli, ${ }^{2}$ G \\ Sangamithra, ${ }^{2}$ PNS Prasad ${ }^{2}$ \\ 'Professor and HOD, Department of Neurology, Narayana \\ medical college and hospital, India \\ ${ }^{2}$ Assistant professor, Department of Neurology, Narayana \\ medical college and hospital, India
}

\begin{abstract}
Correspondence: Dr G Sangamithra, DM Neurology,Assistant professor, Department of Neurology, Narayana Medical College, India, Tel 98495568I5,

Email drsangamithrag@narayanamedicalcollege.com
\end{abstract}

Received: January 29, 2019 | Published: March 26, 2019
The assessment of NMS can be done by using the well validated nonmotor symptom questionnaire (NMSQuest). ${ }^{5-7}$ Chaudhuri and associates developed this questionnaire which can be self administered. ${ }^{5}$ It is 30 component questionnaire which can be applied to the patients and the responses would be given as either Yes or No. NMS evaluated by NMSQ are salivation (SAL), decreased taste and smell (TAS/SML), swallowing (SWA), nausea (NAU), constipation (CON), incontinence of urine/bowels (INCON), incomplete emptying of bladder (INCOMP), urgency of bladder (URG), nocturia (NOCT), generalized body pains (PAIN), change in weight (WC), forgetting things (FT), loss of interest in surroundings (LOI), hallucinations (HAL), delusions (DEL), lack of concentration (LOCn), sadness (SAD), anxiety (ANX), loss of interest in sex (LOIS), difficulty in sex (DISx), dizziness (DIZ), falls (FAL), daytime sleepiness (DTS), difficulty to sleep (DifS), talking in sleep (TIS), dreams (DRM), restless legs (RL), leg swelling (LS), increased sweating (SWE), double vision (DV) which will help in the precise evaluation of NMS. The reporting rate of different NMS was 9 to 12 among the PD patients during their clinic visits, when evaluation was done with the NMSQ. ${ }^{5}$ The UPDRS III ${ }^{8,9}$ (motor examination part of UPDRS) is a battery that comprehensively examines the motor system. UPDRS III contains 14 items to examine the motor disability in PD patients. Hoehn and Yahr scale is a simple scale which can be easily applied in categorizing the motor status into five different stages( I-V) depending on the clinical 
severity of the disease. ${ }^{10,11}$ Compared to UPDRS III, HY scale is less complete and doesn't provide details of specific aspects of motor deficit. Though the UPDRS part I and II assess the NMS, all the domains of non motor symptomatology were not clearly defined and the assessment can be incomplete. Addition of NMSQ is essential to these motor scales to assess the NMS in detail.

\section{Aims and objectives}

1) To study the spectrum of NMS in terms of their prevalence and frequency in a South Indian cohort of Parkinson's disease patients

2) To correlate the total number of NMS with motor severity of the disease.

3) To identify the individual non motor symptoms that has significant association with the motor severity of the disease.

\section{Materials and methods}

The study was an observational, cross sectional study conducted at Narayana medical college and hospital during a period of one year from January 2015 to May 2016. All the patients attending the Neurology OPD with features suggestive of PD and meeting the UK Parkinson's Disease Society Brain Bank clinical diagnostic criteria ${ }^{12}$ were included in the study after obtaining the informed consent from them.

All patients who were suggestive of secondary Parkinsonism and Parkinson plus syndromes were excluded from the study group. The study had been approved by the institutional ethical committee. NMS were assessed by using the NMS Quest. Motor severity was assessed by using the (UPDRS III) motor examination part of UPDRS. All the patients were categorized to the different stages of Hoehn and Yahr staging based on the clinical severity of presentation. All the necessary permissions were taken for the utilization of the above mentioned scales in our study.

\section{Statistical analysis}

SPSS version 20 was used to analyze the study data. Statistical significance between the total NMSQ score, UPDRS III score and H\&Y stages was evaluated using the Pearson's correlation method. The association of individual non motor symptoms with motor severity of the disease was evaluated with the independent $\mathrm{T}$ test for statistical significance. A P value $<0.05$ was taken as significant.

\section{Results}

The study enrolled a total of 45 patients. NMSQuest was administered to all the study participants. Motor examination by UPDRS III was conducted in all the 45 patients. The demographical and clinical variables of the study population were studied (Table 1). Male patients constitute $80 \%$ with a male to female ratio of $4: 1$. Average age of the patients was 64.7 years. Alcoholics were $3(6.7 \%)$ and smokers were $7(15.6 \%) .11(24.4 \%)$ patients had diabetes and $14(31.1 \%)$ patients were hypertensives. Mean NMS total score was $9.29 \pm 4.26$

Number of patients observed in different H\&Y stages were, 19 patients with stage II followed by 10 patients with stage III and 8 patients with Stage I. H\&Y stage 4 patients were 5 and stage V patients were 3. The average number of NMS was 13.3 in H\&Y stage V patients, 11.2 in stage IV patients, 11.3 in stage III patients, 7.6 in stage II patients and 7.8 in stage I patients (Figure 1).
Table I Demographic factors and clinical variables among the cohort of Parkinson's disease patients.

\begin{tabular}{ll}
\hline Variable & Prevalence(\%)N=45 \\
\hline Average Age at examination & $64.7 \mathrm{yrs}$ \\
Sex & $36(80 \%)$ \\
Hypertension & $9(20 \%)$ \\
Diabetes & $14(31.1 \%)$ \\
Smoking & $\mathrm{I} \mathrm{I}(24.4 \%)$ \\
Alcoholism & $3(6.7 \%)$ \\
Total symptoms(NMSQ) & $7(15.6 \%)$ \\
UPDRS III SCORE(0-I08) & $9.29 \pm 4.26$ \\
Hoehn\&Yahr STAGE & $44.2 \pm 9.3$ \\
H\&Y stage I & $2.47 \pm 1.1$ \\
H\&Y stage II & $8(17.7 \%)$ \\
H\&Y stage III & $19(42.2 \%)$ \\
H\&Y stage IV & $10(22.2 \%)$ \\
H\&Y satge V & $5(I 1.1 \%)$ \\
\hline
\end{tabular}

Mean value of UPDRS III score was $44.2 \pm 19.3$. The mean value of $\mathrm{H} \& \mathrm{Y}$ stage was $2.47 \pm 1.1$. Pearson statistical correlation method was used to study the correlation between the scores of NMSQ, UPDRS III and H\&Y staging (Table 2). NMSQ total score had significant statistical correlation with UPDRS III scores suggesting the number of nonmotor symptoms will increase with worsening of motor severity. (P-0.019). H\&Y staging also found to be having significant association with NMSQ score (P-0.005).There exists a very high significant statistical correlation between the UPDRS III score and H\&Y staging $(\mathrm{P}<0.0001)$.

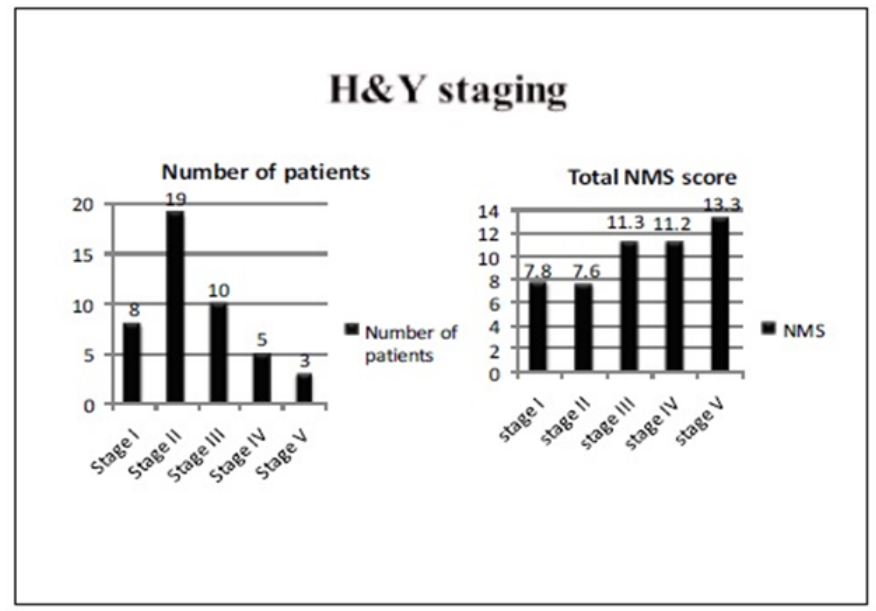

Figure I Distribution of patients and the total NMS score among the different stages of $\mathrm{H} \& Y$ stages.

The prevalence and frequency of different NMS observed in our study were listed in Table 3 and the graphical representation of NMS was done by means of Figure 2. The common non motor symptoms observed were CON (28), ANX (26), URG (25), NOCT (25), SAD(22), and PAIN (20).Least observed symptoms were TIS (1), DV (2), RL (4), and DEL (2) and HAL (3). We also evaluated the statistical correlation for each of the individual nonmotor symptoms to the UPDRS III score and H\&Y staging by using the independent T test (Table 4). INCOMP, DISx, DifS and LS were found to have 
significant correlation with both UPDRS III score and H\&Y staging. The non motor symptom of PAIN found to have statistical correlation only with UPDRS III, where as symptoms like FAL and DV were having significant association with $\mathrm{H} \& \mathrm{Y}$ staging.

Table 2 Pearson correlation between NMS \&UPDRS III, HY staging

\begin{tabular}{llll}
\hline & & $\begin{array}{l}\text { UPDRS Score III } \\
\text { (Motor System) }\end{array}$ & $\begin{array}{l}\text { H \&Y } \\
\text { STAGE }\end{array}$ \\
\hline $\begin{array}{l}\text { Total symptoms } \\
\text { (NMSQ-T) }\end{array}$ & Pearson Correlation & 0.347 & 0.414 \\
& P value & 0.019 & 0.005 \\
\hline
\end{tabular}

NMSQ-T, Non motor symptom questionnaire - Total score, $\mathrm{P}$ value $<0.05$ *significant

Table 3 Prevalence of Nonmotor symptoms among the cohort of Parkinson's disease patients based on NMSQ

\begin{tabular}{|c|c|c|}
\hline Non motor symptoms & Number of patients & Prevalence \\
\hline Saliva & 11 & 24.4 \\
\hline Taste/smell & 11 & 24.4 \\
\hline Swallowing & 7 & 15.6 \\
\hline Nausea & 4 & 8.9 \\
\hline Constipation & 28 & 62.2 \\
\hline Incontinence & 11 & 24.4 \\
\hline Incomplete emptying & 16 & 35.6 \\
\hline Urgency & 24 & 53.3 \\
\hline Nocturia & 23 & 51.1 \\
\hline Pains & 20 & 44.4 \\
\hline Weight change & 11 & 24.4 \\
\hline Forgetting things & 10 & 22.2 \\
\hline Loss of interest & 10 & 22.2 \\
\hline Hallucinations & 4 & 8.9 \\
\hline Delusions & 2 & 4.4 \\
\hline Lack of concentration & 8 & 17.8 \\
\hline Sadness & 22 & 48.9 \\
\hline Anxiety & 26 & 57.8 \\
\hline Loss of Interest in sex & 11 & 24.4 \\
\hline Difficulty in sex & 14 & 31.1 \\
\hline Dizziness & 21 & 46.7 \\
\hline Falls & 12 & 26.7 \\
\hline Day time sleep & 11 & 24.4 \\
\hline Difficulty to sleep & 17 & 37.8 \\
\hline Dreams & 12 & 26.7 \\
\hline Talking in sleep & 1 & 2.2 \\
\hline Restless legs & 4 & 8.9 \\
\hline Leg swelling & 13 & 28.9 \\
\hline Sweating & 8 & 17.8 \\
\hline Double vision & 2 & 4.4 \\
\hline
\end{tabular}

Table 4 Independent samples T-test for statistical significance of individual non motor symptoms with the UPDRS III and HY staging

\begin{tabular}{|c|c|c|}
\hline $\begin{array}{l}\text { Non motor } \\
\text { symptoms mean } \\
(\mathrm{n} / 45)\end{array}$ & $\begin{array}{l}\text { UPDRS III Mean } \\
\text { score }(44.2 \pm 9.3) \\
P \text { value }\end{array}$ & $\begin{array}{l}\text { H\&Y staging Mean } \\
\text { score }(2.2 \pm 1.1) \\
P \text { value }\end{array}$ \\
\hline Saliva & 0.712 & 0.515 \\
\hline Taste/smell & 0.943 & 0.968 \\
\hline Swallowing & 0.435 & 0.173 \\
\hline Nausea & 0.406 & 0.183 \\
\hline Constipation & 0.437 & 0.427 \\
\hline Incontinence & 0.103 & $0.013^{*}$ \\
\hline Incomplete emptying & $0.043^{*}$ & $0.016^{*}$ \\
\hline Urgency & 0.205 & 0.204 \\
\hline Nocturia & 0.786 & 0.163 \\
\hline Pains & $0.026^{*}$ & 0.332 \\
\hline Weight change & 0.634 & 0.235 \\
\hline Forgetting things & 0.63 & 0.461 \\
\hline Loss of interest & 0.208 & 0.291 \\
\hline Hallucinations & 0.791 & 0.69 \\
\hline Delusions & 0.243 & 0.497 \\
\hline $\begin{array}{l}\text { Lack of } \\
\text { concentration }\end{array}$ & 0.526 & 0.436 \\
\hline Sadness & 0.869 & 0.473 \\
\hline Anxiety & 0.843 & 0.405 \\
\hline Interest in sex & 0.064 & 0.072 \\
\hline Difficulty in sex & $0.009 *$ & $0.003 *$ \\
\hline Dizziness & 0.5 & 0.286 \\
\hline Falls & 0.43 & $0.001 *$ \\
\hline Day time sleep & 0.24 & 0.069 \\
\hline Difficulty to sleep & $0.003^{*}$ & $0.025^{*}$ \\
\hline Dreams & 0.665 & 0.633 \\
\hline Talking in sleep & 0.826 & 0.628 \\
\hline Restless legs & 0.788 & 0.951 \\
\hline Leg swelling & $0.001 *$ & $0.003 *$ \\
\hline Sweating & 0.939 & 0.347 \\
\hline Double vision & 0.214 & $0.007 *$ \\
\hline
\end{tabular}

$\mathrm{P}$ value $<0.05 *$ significant Values

\section{Discussion}

NMS are observed universally in all patients with PD at all stages and age groups. But NMS are still under reported by the patients and under recognized and addressed inadequately by the treating physicians as well. Recent international Studies had shown that up to $65.2 \%$ of NMS in PD were under recognized by physicians and nurses. Causative factors for underreporting from the patients were embarrassment in revealing the symptoms as well as unawareness of the patients that these NMS were linked to PD. ${ }^{13}$ 


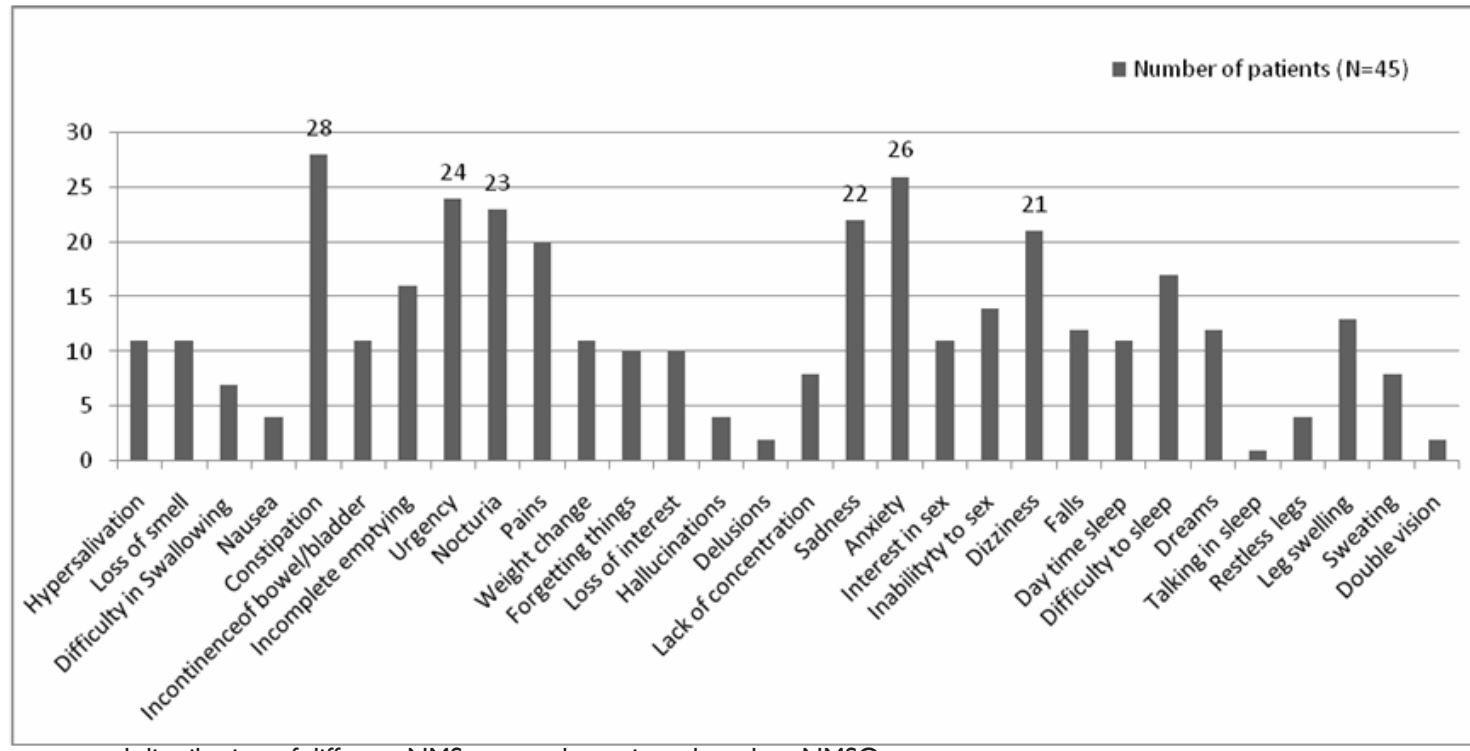

Figure 2 Frequency and distribution of different NMS among the patients based on NMSQ.

Out of 45 patients studied males were the dominant sex in our study, similar to previous studies..$^{14}$ Prevalence rate of diabetes in our study was $11 / 45$ and hypertension was $14 / 45$. The total number of non motor symptoms was ranging from $0-18$. Mean NMS value was of 9.29 with a standard deviation of 4.26 symptoms. The value was little lower compared to the previous multicentre studies conducted at UK, USA, Italy, Japan and Israel where the total NMS was 10.3 with a standard deviation of 5.4. The average of total NMS was more for women being 10.5 compared to men for whom the value was 8.922 . The mean number of NMS reported in PIRAMO study ${ }^{15}$ was 7.8 per patient. A median number of 8 NMS was reported per patient in a study conducted by David et al. among the Belgian population. ${ }^{16}$

In our study group only one patient $(2.2 \%)$ had no non motor symptoms. This was similar to the multicenter survey, the PIRAMO study which was conducted among 1072 patients with PD and the prevalence of NMS was $98.6 \%$. A Chinese cross sectional study conducted by Li et al. in 82 patients with PD found the prevalence of NMS was $100 \%{ }^{17}$

The most common non motor symptoms we observed in our study, in the order of decreasing frequency were CON, ANX, URG, NOCT, SAD, DIZ and PAIN. Most of the previous studies had reported the most frequent NMS were related to the urinary, sleep and sexual disturbances when assessed with the help of NMSQ. These large multicenter studies reported the most common NMS was being NOCT (61\%). The PIRAMO study reported that the most common NMS were ANX, fatigue, DifS and urinary disturbances. Chaudhuri et al. ${ }^{13}$ reported that the most common NMS were urinary disturbances, CON, mood and sleep related. A study conducted by Ravan et al. ${ }^{18}$ observed the urinary problems, CON, sleep and mood related complaints were common in the order of decreasing frequency among the Indian population. The results of our study were compared with previous studies and represented in Table 5. Our study results had a little variation from the previous reports in being CON was the commonest followed by the mood and urinary disturbances. The variation in our study could be attributed to the under reporting of the sleep related problems, and social as well as cultural factors might resulted in lesser reporting of symptoms especially related to the sexual problems. We studied the NMS correlation with the motor symptoms evaluated by both the well renowned methods of motor examination in patients with PD to emphasize the significant association of non motor symptoms with motor severity.

We analyzed the motor symptoms and categorized the patients accordingly in different HY stages. The frequency of NMS across the different HY stages were indicated in Table 2. The mean HY staging was $2.47 \pm 1.1$. Our study found $42 \%$ of patients in HY stage II, $22 \%$ in stage III, $17.7 \%$ in stage I, $10 \%$ in stage IV and $6.6 \%$ in stage V. More advanced disease had a higher HY stage and associated with a higher number of reported NMS. NMSQ total score had significant statistical correlation with the HY scale staging ( p- 0.005). David et al. reported a higher total number of NMS in severe Hoehn and Yahr stages among the Belgian population. ${ }^{16}$

All the patients were examined with UPDRS III to evaluate the motor system more comprehensively. The maximum score was 84 with a mean score of 44.2 with a SD of 19.3. Ravan et al. ${ }^{18}$ analysed UPDRS score and the mean score was 22.8. Factors for higher scores in our patient group could be the severe motor involvement, delayed presentation, with poor symptom control and inadequate treatment. The data analysis revealed significant statistical correlation between the UPDRS III score and total score of NMSQ (p - 0.019). This suggests that with the increase in severity of motor symptoms the non motor symptoms frequency will also increase accordingly.

We also analyzed the data to correlate the individual non motor symptoms to the UPDRS III score and HY scale staging. Urinary disturbances like INCOMP, sexual problems like DISx, sleep related problems like DifS and LS were found to had significant statistical correlation with both UPDRS III score and HY staging among the all NMS. Among the other NMS, FAL and DV were having significant association with $\mathrm{H} \& Y$ staging. PAIN found to had statistical correlation only with UPDRS III.

The study is different from the previous studies in being it was conducted in the south Indian cohort of Parkinson's disease patients. There could be possible existence of geographical and racial differences in the manifestation of NMS compared to the rest of the 
country, which were assessed by our study. Our study also evaluated the significance of individual non motor symptom association with UPDRS III and HY staging which also differentiates it from the rest of the studies.

The limitations of our study were that we failed to gather the treatment details of the patients. Patients on anti parkinsonian medications were reported to be having more number of non motor symptoms and tend to have fluctuations in their NMS akin to motor symptoms. ${ }^{19}$ Being a cross sectional study, we could not rule out the contribution of other comorbidities to the genesis NMS along with the Parkinson's disease. Long term follow ups are required to further establish the association between the studied variables. Though our study population was of good size with regards to the data collection from a single center, the sample size was still inadequate to draw the definite conclusions. Nevertheless our study made an effort to screen the non motor symptoms in systemic way and studied the motor severity of the entire study population with both the UPDRS III and HY scale. We correlated statistically the non motor and motor symptoms and found significant correlation. We also made efforts to correlate each of the non motor symptoms to the UPDRS and HY scale stages.

Table 5 Comparison of our study with previous studies

\begin{tabular}{|c|c|c|c|c|c|c|}
\hline S.no & Author & Year & $\begin{array}{l}\text { Number of } \\
\text { patients }\end{array}$ & $\begin{array}{l}\text { Mean NMS } \\
\text { score }\end{array}$ & $\begin{array}{l}\text { Mean UPDRS } \\
\text { III score }\end{array}$ & Most common NMS \\
\hline I & Ning Zhang et al. & 2015 & 493 & 3.64 & 24.69 & Memory problems \\
\hline 2 & David crosier et al & 2012 & 215 & 8 & 25 & Nocturia \\
\hline 3 & Barone $\mathrm{P}$ et al. & The PRIAMO study 2009 & 1072 & 7.8 & - & Fatigue\& anxiety \\
\hline 4 & Martinez- Martin et al. & 2007 & 545 & 10.3 & - & Urinary symptoms \\
\hline 5 & Amruta Ravan et al. & 2015 & 81 & - & 22.8 & Nocturia, \\
\hline 6 & Our study* & 2016 & 45 & 9.2 & 44.2 & $\begin{array}{l}\text { Constipation, anxiety, } \\
\text { Urinary Urgency }\end{array}$ \\
\hline
\end{tabular}

\section{Conclusion}

This study confirms the high prevalence of NMS in PD patients. CON, ANX, URG were the commonest NMS among the south Indian cohort of Parkinson's disease patients. Number of NMS will increase with increase in the severity of the motor symptoms. INCOMP, DISx, DifS and LS had significant association with UPDRS III and HY staging compared to the rest of the NMS. Awareness of the NMS is essential among the health care providers as the symptoms may not be always offered by the patient and leading to miss the early diagnosis of the condition and sometimes to misdiagnosis. This results in increasing the disease burden to the patient and care givers escalating the health expenditure to the society. NMSQ is a useful clinical tool that will help to screen all the domains of non motor symptoms.

\section{Funding}

There were no sources of funding for the conduction of our study.

\section{Acknowledgments}

We thank the faculty and staff members of department of neurology for collecting the data and the department of statistical analysis and the patients for their cooperation.

\section{Conflicts of interest}

The authors had no conflicts of interest.

\section{References}

1. O'Sullivan SS, Williams DR, Gallagher DA, et al. Nonmotor symptoms as presenting complaints in Parkinson's disease: a clinicopathological study. Mov Disord. 2008; 23(1):101-106.

2. Chaudhuri KR, Schapira AH. Non-motor symptoms of Parkinson's disease: dopaminergic pathophysiology and treatment. Lancet Neurol. 2009;8:464-474.

3. Kempster PA, O’Sullivan SS, Holton JL, et al. Relationships between age and late progression of Parkinson's disease: a clinico-pathological study. Brain. 2010;133(Pt 6):1755-1762.

4. Wickremaratchi MM, Ben-Shlomo Y, Morris HR. The effect of onset age on the clinical features of Parkinson's disease. Eur J Neurol. 2009; $16(4): 450-456$.

5. Chaudhuri KR, Martinez-Martin P, Schapira AH, et al. International multicenter pilot study of the first comprehensive self-completed nonmotor symptoms questionnaire for Parkinson's disease: The NMS Quest study. Mov Disord. 2006;21(7):916-923.

6. Martinez-Martin P, Schapira AH, Stocchi, et al. Prevalence of nonmotor symptoms in Parkinson's disease in an international setting; Study using nonmotor symptoms questionnaire in 545 patients. Mov Disord. 2007;22(11):1623-1629.

7. Romenets SR, Wolfson C, Galatas C et al. Validation of the non-motor symptoms questionnaire (NMS-Quest). Parkinsonism Relat Disord. 2012;18(1):54-58.

8. Movement Disorder Society Task Force on Rating Scales for Parkinson's, Disease. The Unified Parkinson's Disease Rating Scale (UPDRS): status and recommendations. Movement disorders: official journal of the Movement Disorder Society. 2003;18(7):738-750.

9. Goetz CG, Fahn S, Martinez-Martin P, et al. Movement Disorder Society-sponsored revision of the Unified Parkinson's Disease Rating Scale (MDS-UPDRS): Process, format, and clinimetric testing plan. Mov Disord. 2007;22 (1):41-47.

10. Hoehn M, Yahr M. Parkinsonism: onset, progression and mortality. Neurology. 1967;17 (5):427-442.

11. Goetz CG, Poewe W, Rascol O, et al. Movement Disorder Society Task Force report on the Hoehn and Yahr staging scale: status and recommendations. Mov Disord. 2004;19(9):1020-1028.

12. Hughes AJ, Daniel SE, Kilford L, et al. Accuracy of clinical diagnosis of idiopathic Parkinson's disease. A clinico-pathological study of 100 cases. J Neurol Neurosurg Psychiatry. 1992;55(3):181-184.

13. Chaudhuri KR, Prieto-Jurcynska C, Naidu Y, et al. The nondeclaration of nonmotor symptoms of Parkinson's disease to health care professionals: 
An international study using the nonmotor symptoms questionnaire. Mov Disord. 2010;25(6):704-709.

14. Song Y, Gu Z, An J, et al. Gender differences on motor and non-motor symptoms of de novo patients with early Parkinson's disease. Neuro Sci. 2014;35(12):1991-1996.

15. Barone P, Antonini A, Colosimo C, et al. PRIAMO study group. The PRIAMO study: A multicenter assessment of nonmotor symptoms and their impact on quality of life in Parkinson's disease. Mov Disord. 2009;24(11):1641-1649.

16. David Crosiers, Barbara Pickut, Jessie Theuns, et al. Non-motor symptoms in a Flanders-Belgian population of 215 Parkinson's disease patients as assessed by the Non-Motor Symptoms Questionnaire. Am J Neurodegener Dis. 2012;1(2):160-167.

17. Wang G, Hong Z, Cheng Q, et al. Validation of the Chinese non-motor symptoms scale for Parkinson's disease: Results from a Chinese pilot study. Clin Neurol Neurosurg. 2009;111(6):523-526.

18. Ravan A, Ahmad FM, Chabria S, et al. Non-motor symptoms in an Indian cohort of Parkinson's disease patients and correlation of progression of non-motor symptoms with motor worsening. Neurol India. 2015;63(2):166-174.

19. Witjas T, Kaphan E, Azulay JPet al. Nonmotor fluctuations in Parkinson's disease: frequent and disabling. Neurology. 2002;59(3):408-413. 